\title{
LAS POLÍTICAS DE EVALUACIÓN DE LA ACTIVIDAD INVESTIGADORA Y DOCENTE EN LAS AGENCIAS AUTONÓMICAS DE EVALUACIÓN EN ESPAÑA: UN ANÁLISIS COMPARATIVO
}

Natalia Quintas-Froufe'1: Universidade da Coruña. España $\underline{\text { n.quintas.froufe@udc.es }}$

\section{RESUMEN}

El artículo que aquí se propone tiene como objetivo analizar los criterios, directrices y baremos que las distintas agencias autonómicas de evaluación han adoptado a la hora de valorar los méritos de carácter docente e investigador de los solicitantes para lograr la evaluación positiva en la figura de contratado doctor en el campo de las Ciencias Sociales y Jurídicas, y más concretamente en el área de Comunicación Audiovisual y Publicidad. Para ello se consultaron los criterios recogidos en cada una de las diez Agencias Autonómicas de Evaluación españolas: AGAE, ACPUA, AQU Catalunya, AQUIB, ACECAU, ACSUCYL, ACSUG, ACAP, UNIBASQ y AVAP.

PALABRAS CLAVE: Agencias de evaluación - Acreditación - Universidad 


\title{
POLICIES FOR THE EVALUATION OF RESEARCH AND TEACHING USED BY SPAIN'S AUTONOMOUS EVALUATION AGENCIES: A COMPARATIVE ANALYSIS
}

\begin{abstract}
The article proposed here in has the aim of analyzing the criteria, directives and standards which the various autonomous evaluation agencies have adopted for the assessment of the merits of a candidate's educational and research career to allow a positive evaluation in the selection process for the post of Doctor in the field of Social Sciences and Law, specifically relating to the area of Audiovisual Communication and Advertising. To this end, the criteria used by each of the ten Autonomous Evaluation Agencies in Spain were referenced: AGAE, ACPUA, AQU Catalunya, AQUIB, ACECAU, ACSUCYL, ACSUG, ACAP, UNIBASQ and AVAP.
\end{abstract}

KEY WORDS: Evaluation agencies - Evaluation - University

\section{INTRODUCCIÓN}

La entrada en vigor de la Ley Orgánica 6/2001 (LOU), de 21 de diciembre, de Universidades (modificada posteriormente por la Ley Orgánica 4/2007, de 12 de abril) supuso un nuevo orden en el ámbito universitario y en las políticas de evaluación del profesorado. La creación de nuevas categorías contractuales y de mecanismos externos de evaluación de la propia actividad universitaria y del profesorado originó una profunda transformación del sistema universitario español con el fin último de la mejora de calidad académica.

Uno de los mecanismos que se pusieron en marcha fue la creación de la Agencia Nacional de Evaluación de la Calidad y la Investigación $\left(\mathrm{ANECA}^{1}\right)$. Los orígenes de la fundación de la ANECA se remontan al año 2002 cuando, tras el acuerdo del Consejo de Ministros del día 19 de julio de 2002, se autoriza al Ministerio de Educación, Cultura y Deporte su creación.

El espíritu que subyace bajo dicha fundación está recogido en la propia ley, en la cual se vincula su constitución con la necesidad de "mejorar la calidad de la docencia y la investigación, a través de un nuevo sistema objetivo y transparente, que garantice el mérito y la capacidad en la selección y el acceso del profesorado, y mejorar, 
Por otra parte, se crearon nuevas figuras académicas al amparo de la ley como la categoría de profesor contratado doctor. La finalidad del contrato de profesor/a contratado doctor era "desarrollar, con plena capacidad docente e investigadora, tareas de docencia y de investigación, o prioritariamente de investigación" (LOU, 2001, p.49413). Para poder acceder a dicha figura era preciso poseer el título de doctor, la acreditación de al menos tres años de actividad docente e investigadora, o

A partir de ahora ANECA prioritariamente investigadora, postdoctoral ${ }^{2}$ y la evaluación positiva por parte de la Agencia Nacional de Evaluación de la Calidad y la Investigación o del órgano de evaluación externa que la ley de la Comunidad Autónoma determine, que finalmente fueron las agencias autonómicas de evaluación.

En un principio únicamente ocho comunidades autónomas (Cataluña, Andalucía, Galicia, Castilla y León, Canarias, Comunidad Valenciana, Comunidad de Madrid e Islas Baleares) contaban con su propia agencia de evaluación. En la actualidad nos encontramos con diez agencias de evaluación pertenecientes a diez Comunidades Autónomas, objeto de nuestro estudio.

Al margen de otras funciones administrativas y de carácter institucional, las agencias de evaluación autonómicas desempeñan una actividad básica en relación al profesorado universitario: "acreditar la calidad de la investigación y la experiencia investigadora del evaluado" (De las Heras y Polo, 2009: 63), función determinante para esta investigación.

Por consiguiente, la evaluación del profesorado universitario por parte de un órgano ajeno a la misma fue una de las principales innovaciones de la ley y derivó en que la ANECA y las agencias autonómicas de evaluación se convirtieron en organismos claves para la carrera académica del profesorado universitario al establecer las políticas de evaluación de su actividad que permitirían su promoción.

Si bien dichas agencias autonómicas se agruparon en el año 2006 formando la Red Española de Agencias de Calidad Universitaria (REACU) con el fin de "promover y desarrollar la cooperación y el intercambio de experiencias e información, especialmente de metodologías y buenas prácticas" (Acuerdo Constitutivo de la REACU, 2006) nos interesa averiguar si dicho espíritu cooperativo se traslada al ámbito de las políticas de evaluación del profesorado. 


\section{METODOLOGÍA}

El objeto de estudio de esta investigación son las políticas de evaluación de la docencia y la investigación que han adoptado las agencias de evaluación autonómicas en España con el fin de analizar las principales directrices que rigen el proceso evaluador.

La selección de dicho objeto vino determinado por la preeminencia de dichos ámbitos en la carrera académica. La docencia y la investigación son dos de las actividades que mayor peso tienen en el currículum del profesor universitario (al margen de otras como la gestión o la experiencia profesional), y consecuentemente, son las determinantes a la hora de conseguir una acreditación positiva.

Para estudiar dichas políticas analizaremos los criterios seguidos por cada de las agencias en el área de Ciencias Sociales y Jurídicas para la evaluación positiva en la modalidad contractual de profesor contratado doctor, al ser una figura laboral de carácter estable.

La información necesaria para llevar a cabo esta investigación se obtuvo de las páginas web de las agencias visitadas con frecuencia durante todo el proceso de recogida de datos y posterior análisis, en donde están expuestos los criterios de evaluación que utilizan. Se tomaron como referencia las últimas versiones de dichos documentos que datan de diferentes años en función de su actualización. En algunos casos fue necesario ponerse en contacto con algunas agencias para corroborar la veracidad de ciertos datos.

Por tanto, se propone una investigación de carácter eminentemente descriptivo de las políticas de evaluación de las agencias autonómicas en la que se exponen los apartados del currículum del solicitante establecidos y los criterios de valoración de los méritos, así como las recomendaciones que se realizan a los demandantes de dicha evaluación sobre la interpretación de dichos baremos. También analizaremos las puntuaciones máximas y mínimas exigidas en cada uno de los apartados destinados a la evaluación de la experiencia docente e investigadora establecidos por las diferentes agencias.

A continuación expondremos los criterios publicados por cada una de las diez agencias para finalizar con las principales conclusiones en las que se señalen las diferencias y similitudes detectadas durante el proceso de análisis. 


\section{ANÁLISIS Y DISCUSIÓN}

\subsection{La Agencia Andaluza de Evaluación (AGAE) ${ }^{3}$}

La valoración de los méritos que la Agencia Andaluza de Evaluación realiza parte de cuatro dimensiones: (1) Experiencia investigadora y transferencia del conocimiento (50 puntos sobre 100), (2) Experiencia docente (40 puntos sobre 100), (3) Formación académica (8 puntos sobre 100), y (4) Otros méritos (2 puntos sobre 100), siendo la experiencia investigadora del solicitante lo más valorado de su currículum.

La AGAE establece que la puntuación mínima a alcanzar para obtener una acreditación positiva es de 55 puntos. No obstante, debe cumplirse un prerrequisito previo: alcanzar 50 puntos en la suma de los apartados (1) y (2) obteniendo un mínimo de 20 puntos en la experiencia investigadora y un mínimo de 15 en la experiencia docente para que pueda efectuarse la suma de todos los apartados. Veamos cuáles son los méritos valorados en ambos apartados.

Con respecto a la primera dimensión (1) Experiencia investigadora y transferencia del conocimiento, la clasificación de los méritos se realiza en función de siete parámetros, que expondremos a continuación. Merece ser destacada la orientación indicada sobre la experiencia investigadora "se considerará suficiente experiencia investigadora una similar a la que produce la evaluación positiva de un tramo de investigación por la Comisión Nacional de Evaluación de la Actividad Investigadora (CNEAI) en cada campo correspondiente". También se hace referencia a que se tendrán en cuenta los méritos de los últimos cuatro años.

Para la valoración del primer parámetro, (1.1) Publicaciones científicas con proceso anónimo de revisión por pares y patentes en explotación, se discierne entre revistas indexadas 4 / no indexadas ${ }^{5}$ y se enumeran los índices considerados en el campo de las Ciencias Sociales («Social Sciences Citation Index, el Science Citation Index u otros listados similares generalmente admitidos en este ámbito). Se concreta que otras bases de datos internacionales, relacionadas con un campo del saber determinado,

\footnotetext{
${ }^{3}$ Resolución de 26 de julio de 2005, de la Dirección General de Universidades, por la que se establecen los criterios de acreditación para las figuras contractuales de Profesorado Universitario (Boletín Oficial de la Junta de Andalucía, 8 de agosto 2005, núm. 153, p.7-9).

${ }^{4}$ Valoradas en función de "el lugar que ocupa la revista en el conjunto de las que corresponden a un mismo ámbito de conocimiento, el índice de impacto, el número de autores, la posición que ocupe entre ellos el solicitante, el tiempo transcurrido desde la lectura de la tesis doctoral y la coherencia de una línea de investigación bien definida y mantenida a lo largo del tiempo; no obstante, los cambios a nuevas líneas, con resultados satisfactorios, serán considerados positivamente".
} 
también pueden ser consideradas como índices de calidad. Resulta llamativa la mención explícita a las revistas electrónicas que se realiza, valoradas únicamente si forman parte de los listados del Institute for Scientific Information.

Para obtener la puntuación máxima en este apartado en las áreas de Ciencias de la Educación, Ciencias de la Comunicación y Periodismo, Sociología, Ciencias Políticas y Ciencias de la Administración "se considera necesario, a título orientativo, la publicación de, al menos, 2-4 artículos, en función de su calidad, en revistas recogidas en los listados mencionados anteriormente y 3 a 6 artículos, en función de su calidad, publicados en revistas no indexadas que cumplan los requisitos señalados anteriormente". Se puntualiza también que se podrán tener en cuenta un número menor de trabajos en función de la calidad de la publicación en el área de conocimiento.

Los criterios citados en el segundo parámetro (1.2) Libros y capítulos de libros, hacen referencia a la valoración preferente de la publicación en editoriales especializadas de reconocido prestigio donde "pueda garantizar un riguroso proceso de selección y evaluación de los originales", no obstante, no se hace mención explícita a ninguna de ellas. Se considera que la calidad de dichos méritos vendrá determinada por "el número de citas recibidas de otros autores, el prestigio de la editorial, los editores, la colección en la que se publica la obra, las reseñas en las revistas científicas especializadas, su difusión, su extensión y las traducciones a otras lengua".

Por otra parte, se hacen dos observaciones significativas. La primera de ellas alude a la publicación monográfica de la tesis doctoral, que no se evaluará, a no ser que "haya experimentado una profunda revisión y remodelación con posterioridad a su defensa pública"; mientras que la segunda advierte de la no consideración de las actas de congreso como capítulos de libro aunque éstas sean publicadas con ISBN.

La tercera dimensión es específica de los (1.3) Proyectos de investigación. Para su valoración se tendrá en cuenta el grado de responsabilidad en el proyecto y la tipología del mismo (europeo, nacional, autonómico, público/privado o sometido a evaluación externa de la Agencia Nacional de Evaluación y Prospectiva).

En relación al cuarto apartado, (1.4) Resultados de la investigación, se determina que será la "aplicada a la solución de problemas relevantes del ámbito cultural, social, técnico, económico y empresarial y que contribuyan a la innovación del tejido económico y social". 
La (1.5) Dirección de tesis doctorales será el quinto apartado de evaluación. Éstas serán valoradas en función de "la mención de doctorado europeo, la mención de calidad del programa de doctorado, la calidad de los trabajos publicados relacionados con la misma y el prestigio de los centros en los que se ha llevado a cabo". Se puntualiza que también se consideran, con menor peso, las tesis en ejecución 6 .

Con respecto a las (1.6) Contribuciones presentadas en congresos, conferencias, seminarios $u$ otros tipos de reuniones de relevancia científica únicamente se tienen en cuenta los que tienen procedimientos selectivos en la admisión. El tipo de participación y el carácter nacional/internacional, junto con otros aspectos relativos al área de conocimiento serán determinantes. Por último, en el apartado de (1.7) Otros méritos de investigación, se engloban todos los méritos que no tuvieran cabida en ninguna de las secciones anteriores.

A la hora de valorar la experiencia docente del solicitante, se determinan cinco apartados de evaluación. En el primero de ellos, (2.1) Amplitud, intensidad, diversidad, grado de responsabilidad, ciclos y tipo de docencia en su ámbito disciplinar universitario en enseñanzas regladas y no regladas, se establece un número de horas mínimo, de carácter orientativo, para alcanzar la máxima puntuación (600 horas de docencia).

Los criterios de calidad indicados se recogen en la propia denominación del apartado además de tener en cuenta la naturaleza y el número de asignaturas impartidas, la coordinación de las mismas, las instituciones nacionales o extranjeras en las que se ha ejercido la docencia, especialmente la desarrollada en centros diferentes al que se defendió la tesis doctoral. Se incluye también en este apartado la dirección o la participación en enseñanzas de postgrado oficial.

El segundo apartado juzga la calidad de la docencia en función de las evaluaciones aportadas (2) Evaluaciones sobre la calidad de su docencia que aporte el solicitante), dándole una mayor relevancia a "las relacionadas con la innovación docente y sus resultados en términos del impacto en el proceso de aprendizaje". En el siguiente apartado, (3) Actuación como ponente en seminarios y cursos, y participación en congresos específicamente orientados a la formación para la actividad docente universitaria se valora, sobre todo, las ponencias por invitación.

Por último, los méritos relacionados con el material docente y la innovación se clasifican en dos apartados: (4) Material docente original cualquiera que sea el soporte utilizado y publicaciones relacionadas con la docencia y (5) Desarrollo de 
instrumentos y herramientas de innovación docente, basadas en la utilización de las TICs y metodologías avanzadas de aprendizaje.

En el primero se tendrá en cuenta "el material docente original desarrollado por el solicitante, cualquiera que sea el soporte utilizado, así como las publicaciones (libros y artículos) relacionadas con la docencia, valorando especialmente su carácter innovador", y también la participación en proyectos de innovación docente y planes/equipos de trabajo relacionados con el Espacio Europeo de Educación Superior, "especialmente en relación con su adaptación a las competencias necesarias para el aprendizaje del alumno".

En el segundo apartado "se valorará especialmente el material docente publicado en la red, las tutorías virtuales y el contenido de la página web". Merece ser destacado el hecho de que al margen de las puntuaciones globales de cada apartado, dicha agencia nos muestra también las puntuaciones mínimas y máximas que se pueden obtener con cada una de las aportaciones que presente el solicitante.

\subsection{La Agencia de Calidad y Prospectiva Universitaria de Aragón (ACPUA)}

Esta agencia no desarrolla actualmente procesos de evaluación del profesorado universitario, la propia página web remite a la ANECA en el caso de necesitar ser evaluado.

\subsection{L' Agència per a la Qualitat del Sistema Universitari de Catalunya (AQU Catalunya) ${ }^{7}$}

La AQU Catalunya es la institución responsable de evaluar la actividad docente e investigadora del profesorado catalán, no obstante, su función difiere de la del resto de agencias autonómicas ya que está orientada fundamentalmente a la evaluación de la actividad investigadora del profesorado, y no la docente ${ }^{8}$.

Otra particularidad que encontramos en el caso catalán es la existencia de una doble tipología de profesor contratado doctor: el profesor agregado y el catedrático contratado. Únicamente nos referiremos a la figura de profesor agregado, al ser la primera categoría de carácter estable a la que se puede acceder en la carrera académica.

Según la legislación autonómica catalana, los requisitos básicos que un solicitante debe cumplir para formar parte de un concurso de profesor agregado, además del título de doctor, son: a) acreditar un mínimo de tres años de actividad docente y de 
de tecnología o de conocimientos, en situación de desvinculación académica de la universidad convocante ${ }^{9}, \mathrm{y}$, por último, c) disponer de una acreditación de investigación ${ }^{10}$, expedida por la AQU, que es la que analizaremos a continuación (Ley 1/2003, de 19 de febrero, de Universidades de Cataluña).

Los apartados básicos de evaluación de la acreditación de investigación son: (1) Publicaciones y transferencia de los resultados de investigación (70\%), (2) Proyectos (15\%), (3) Actividad formativa (7,5\%) y (4) Otros méritos (7,5\%). Esta agencia no establece mínimos a alcanzar en cada uno de los epígrafes. Cabe destacar la mención explícita y valorable del "grado de creatividad, de diversidad temática y de innovación en la investigación del solicitante".

Todo el peso de la evaluación recae en el primer apartado, con una valoración del $70 \%$ sobre la calificación final. En dicho apartado se distingue entre artículos y libros. La valoración de los artículos girará en torno a la revista en la que fueron publicados. Se menciona el índice ISI (y similares) como referencia de calidad y también se establece una triple clasificación (A, B y C):

A) Revista de ámbito internacional, del máximo nivel de calidad en su disciplina, situada en los índices internacionales con los coeficientes de impacto más elevados.

B.1) Revista de ámbito internacional, situada en los índices internacionales con un coeficiente de impacto inferior a las del grupo A.

B.2) Revista no indexada del máximo nivel de calidad en su ámbito, de alcance internacional, con un sistema de evaluación de originales riguroso y un comité científico internacional.

C) Revista de buen nivel de calidad en su ámbito, con un sistema de evaluación riguroso."

Se recomienda que los solicitantes tengan publicado un mínimo de diez artículos, estando insertados cuatro de ellos en revistas A o B2, aunque se señala que no son mínimos exigibles sino una mera orientación. En relación a los libros se establece una doble tipología en función de si los libros han sido publicados en editoriales internacionales de reconocido prestigio o en editoriales prestigiosas de alcance nacional con criterios de selección rigurosos.

\footnotetext{
9 "Este requisito se considera cumplido si los estudios de doctorado fueron cursados íntegramente en
} 
En cuanto a la transferencia de los resultados se valorarán "aquellos cuyo uso actual pueda ser demostrado por el solicitante, o bien que tengan lo suficientemente avanzado el trámite para la protección internacional de derechos, de tal forma que la comisión pueda evaluar el interés real de la aplicación".

En el segundo apartado, orientado exclusivamente a la valoración de los proyectos, se puntualiza que únicamente serán valorados los financiados en convocatorias competitivas, de las administraciones públicas (europeas, estatales, autonómicas) como del sector privado (fundaciones, empresas...). Otros factores a tener en cuenta son el número de proyectos (uno será el mínimo estipulado), el grado de participación, liderazgo y la relevancia del proyecto.

En el tercer apartado, (3) Actividad formativa, se evalúa la capacidad de formar un grupo de investigación, la dirección tesis doctorales y la implicación en la impartición/coordinación de cursos de doctorado posgrados y másters. Y ya por último, en el cuarto epígrafe (4) Otros méritos, se incluirá todo lo no mencionado en el currículum con anterioridad: "premios y galardones a la investigación, tramos de investigación reconocidos, participación como conferenciantes invitados en congresos o universidades, estancias de investigación, cargos de responsabilidad científica (comités editoriales, comités científicos internacionales y nacionales, organización/presidencia de congresos), informes emitidos por profesionales de reconocido prestigio, actividad institucional de promoción y gestión de investigación, etc". Cabe destacar la inclusión de méritos de gran peso en la carrera académica en dicho apartado, como puede ser un tramo de investigación, uno de los menos valorados en el currículum del solicitante.

\subsection{L' Agència de Qualitat Universitària de les Illes Balears (AQUIB) ${ }^{11}$}

La Agencia de Calidad Universitaria de las Islas Baleares valora los méritos de los solicitantes en función de cuatro epígrafes: (1) Experiencia investigadora (55 puntos sobre 100), (2) Experiencia docente (30 puntos sobre 100), (3) Formación académica (8 puntos sobre 100), (4) Experiencia y otros méritos profesionales (4 puntos sobre 100) y (5) Otros méritos (3 puntos sobre 100). Para conseguir la evaluación positiva es necesario obtener una puntuación de 55 puntos siempre que se alcance previamente la puntuación mínima de 25 puntos en el apartado de la experiencia investigadora. Éstas son las únicas indicaciones sobre los baremos de los méritos de los solicitantes ya que no se establecen otras puntuaciones orientativas sobre las aportaciones del solicitante.

Dentro del epígrafe de evaluación de la experiencia investigadora se señalan nueve 
evaluar las publicaciones científicas. En el primero (1.1) se incluyen aquellas de reconocida calidad en el área de conocimiento. En este caso no se menciona ningún índice sino que se alude a la última convocatoria de la Comisión Nacional de Evaluación de la Actividad Investigadora (CNEAI) para conocer los criterios que se van a aplicar. Se puntualiza también que en la rama de conocimiento de Ciencias Sociales y Jurídicas se debe aportar al menos tres publicaciones para "poder obtener una puntuación elevada". En el segundo apartado (1.2) se incluyen las publicaciones científicas que no cumplen los requisitos anteriores.

Los tres siguientes apartados están relacionados con la (1.3) transferencia de conocimiento (patentes, modelos de utilidad y demás resultados que produzcan transferencia tecnológica en el sector productivo), (1.4) la participación en proyectos y (1.5) los contratos de investigación. Los criterios de valoración de los dos últimos inciden en la evaluación externa de un organismo independiente.

En relación al siguiente apartado, (1.6) Estancias en centros de investigación, se subraya la financiación de las mismas en programas competitivos. Se establece un estándar mínimo de 18 semanas (no necesariamente continuadas) de investigación postdoctoral en centros españoles o extranjeros de reconocido prestigio.

En las (1.7) contribuciones a congresos, conferencias, seminarios o cualquier otro tipo de reunión de relevancia científica se valora dicha contribución y también la organización de cualquiera de estos congresos. Se excluyen de este apartado, salvo excepciones, los cursos de verano y las jornadas profesionales. Los últimos méritos a valorar en este apartado serán (1.8) la dirección de tesis doctorales y (1.9) otros méritos de investigación relevantes.

A la hora de evaluar la experiencia docente del solicitante, los méritos se agrupan en siete apartados. El primero de ellos se corresponde con la propia (2.1) experiencia docente; se prioriza la docencia teórica o teórico-práctica en el ámbito universitario y en las materias de los planes de estudios de las enseñanzas universitarias oficiales. Se tendrá en cuenta también (2.2) las instituciones o centros en los que se ha ejercido la docencia y las evaluaciones de calidad de la misma (2.3). También se valora la (2.4) formación didáctica para la actividad docente, (2.5) la participación en proyectos de innovación didáctica y (2.6) el material docente elaborado (independiente del soporte). Por último, se dejará un apartado para incluir a los otros méritos docentes relevantes (2.7). 


\subsection{La Agencia Canaria de Evaluación de la Calidad y Acreditación Universitaria (ACECAU)}

La legislación autonómica canaria estableció dos modalidades de profesor contratado doctor (tipo $1^{12}$ y $2^{13}$ ) en las que era requisito imprescindible disponer de una evaluación positiva para poder acceder a dicho concurso de acceso. Únicamente nos detendremos en la primera por ser la equiparable a la figura de contratado doctor definida por la LOU al valorarse conjuntamente los méritos correspondientes a la investigación y a la docencia, y no solamente los de investigación como ocurre en la segunda modalidad.

La evaluación del currículum de un profesor contratado doctor tipo 1 se realizará en función de cuatro bloques de méritos valorados sobre un máximo de 100 puntos: (1) Actividad investigadora (45 puntos), (2) Docencia (30 puntos), (3) Formación académica (15 puntos) y (4) Actividad profesional y otros méritos (10 puntos). El primer y el segundo apartado son los determinantes para conseguir la acreditación positiva por la exigencia de mínimos obligatorios en ambos.

En la evaluación de la actividad investigadora es necesario obtener 10 puntos, puntuación que debe ser conseguida tras la suma de dos epígrafes: 1. Publicaciones, estancias, congresos, exposiciones, etc y 2. Congresos. No obstante, no todos los méritos englobados en dichos estos epígrafes van a tenerse en cuenta a la hora de alcanzar dicha puntuación ya que se excluyen algunos de ellos, como por ejemplo los relacionados con las estancias predoctorales/ postdoctorales $^{14}$.

12 “a) Profesor contratado doctor tipo 1, para profesores doctores cuya trayectoria docente y labor investigadora sea positivamente evaluada por la Agencia Canaria de Evaluación de la Calidad y Acreditación Universitaria o por la Agencia Nacional de Evaluación de la Calidad y Acreditación desarrollarán tareas docentes y de investigación". Decreto 140/2002, de 7 de octubre, sobre régimen del personal docente e investigador contratado y sobre complementos retributivos del profesorado de las Universidades canarias (Boletín Oficial de Canarias, núm. 139, viernes 18 de octubre de 2002, pp.17058-17067).

13 "b) Profesor contratado doctor tipo 2, para doctores que reúnan relevantes méritos investigadores positivamente evaluados por la Agencia Canaria de Evaluación de la Calidad y Acreditación Universitaria o por la Agencia Nacional de Evaluación de la Calidad y Acreditación. Sus funciones serán preferentemente investigadoras, con la obligación contractual de dirigir proyectos y equipos de investigación y de someter a evaluación por la Agencia cada seis años los resultados obtenidos." Decreto 140/2002, de 7 de octubre, sobre régimen del personal docente e investigador contratado y sobre complementos retributivos del profesorado de las Universidades canarias (Boletín Oficial de Canarias, núm. 139, viernes 18 de octubre de 2002, pp.17058-17067).

${ }^{14}$ Se valoran diez ítems de los dieciocho posibles: Estancias predoctorales (excluido), estancias postdoctorales (excluido), publicaciones científicas en revista de nivel medio alto de su área, 
En relación a la actividad docente se establece un mínimo de 12 puntos, señalando también aquellos méritos que pueden valorarse para su suma. Para alcanzar el mínimo exigido en la actividad docente se evalúan seis ítems de los dieciséis posibles $^{15}$, los cuales son los relativos a la propia experiencia docente. Se excluye de esta suma, por ejemplo, los méritos correspondientes a la formación docente.

A diferencia de otras agencias de evaluación, la ACECAU expone un listado exhaustivo de méritos (cincuenta ítems generales dentro de los cuales se encuentran sub-apartados) con su correspondiente horquilla de puntos (libro: 3-10 puntos) o la puntuación exacta de cada mérito (dirección de tesis doctorales: 7 puntos/ítem).

La actividad investigadora se juzgará en función decuatro aspectos: (1) Publicaciones, estancias, congresos, exposiciones, (2) Congresos, (3) Participación en proyectos y contratos y (4) Otros méritos. Otra particularidad será que dicha agencia no determinará la puntuación de cada uno de esos apartados, ni tampoco las puntuaciones máximas de cada sub-apartado ${ }^{16}$, sino que establecerá las puntuaciones de cada uno los méritos de modo individual.

En relación a la docencia, ésta será valorada en función de (1) Formación para la docencia (hasta un máximo de 10 puntos), (2) Experiencia docente y (3) Otros méritos.

\subsection{La Agencia para la Calidad del Sistema Universitario de Castilla y León (ACSUCYL)}

La ACSUCYL establece cuatro apartados de evaluación: (1) Formación académica, (2) Actividad docente, (3) Actividad investigadora y (4) Experiencia profesional y otros méritos. La actividad investigadora será la más valorada (40 puntos sobre 100), seguida de la docencia (30 puntos sobre 100), la formación académica (15 puntos sobre 100) y la experiencia profesional y otros méritos (15 puntos sobre 100). Se establece una note de corte a alcanzar en dos de los apartados: en la actividad docente $(10 \text { puntos })^{17}$ y en la investigación (20 puntos) ${ }^{18}$. Para conseguir la acreditación positiva es necesario alcanzar en la suma de todos los apartados 60

\footnotetext{
15 Únicamente se tiene en cuenta la Experiencia docente universitaria en su área o área afín, Experiencia docente universitaria en otras áreas, colaboración como becario, tutor en centros asociados de UNED, experiencia docente no-universitaria, experiencia en cursos de doctorado o especialización.

${ }^{16}$ A excepción de los apartados de congresos y de formación docente en donde sí se especificará un
} 
puntos.

En el epígrafe de (2) la actividad docente los méritos se clasificarán en función de nueve parámetros ${ }^{19}$, de los cuales nos detendremos en los dos de mayor puntuación (actividad docente reglada y actividad no reglada) por ser los que aparecen mencionados explícitamente en el documento de orientaciones del solicitante. Con relación a la actividad docente reglada se fija como norma que el solicitante haya impartido tres cursos académicos de docencia reglada ${ }^{20}$. A la hora de valorar dicha docencia se tiene en cuenta especialmente la actividad docente teórica en planes de estudios oficiales y recogida en el POD (Plan de Ordenación Docente), puntuándola con el doble que la docencia práctica. Otros criterios utilizados para la evaluación son el número de créditos impartidos y la naturaleza de la docencia.

En la actividad docente no reglada se valoran los cursos/conferencias "fuera del ámbito curricular" que puede ser la propia Universidad o fuera de ella. La valoración de esta actividad docente se hace teniendo en cuenta "la coherencia de los cursos impartidos con el CV del solicitante, la categoría de la institución organizadora, el carácter formativo para el ámbito universitario o de posgrado, el carácter de divulgación y la duración de los mismos (número de créditos)". Resulta llamativo que la formación docente no esté incluida en este apartado sino en el de (1) Formación académica.

En la (3) actividad investigadora será básica la productividad del solicitante, "entendida como la relación entre número y calidad de los méritos/años en los que se han generado", siendo el periodo de evaluación mínimo a considerar de seis años. Por ello, se pide al solicitante que indique el momento desde el que quiere ser evaluado. En relación a la actividad investigadora se mencionan nueve parámetros ${ }^{21}$ siendo los determinantes las publicaciones científicas y las patentes, que son a los que le prestaremos atención.

\footnotetext{
${ }^{19}$ Actividad Docente Reglada (POD/Hoja de Servicios) (35 puntos), Actividad no reglada (10 puntos) , Cursos fuera del ámbito curricular o fuera del ámbito universitario (6 puntos), Evaluación de la Actividad Docente Aportada (1 punto), Coordinación Movilidad Estudiantil (2 puntos), Dirección y/o participación en proyectos de innovación educativa (3 puntos), Elaboración de Material Didáctico (2 puntos), Publicaciones de Carácter Pedagógico (3 puntos), Dirección Proyectos Fin de Carrera (2 puntos).

${ }_{20}$ Con carácter excepcional se aceptan solicitudes que no cumplan con este requisito aunque debe estar perfectamente justificado (investigadores de reconocido prestigio que desarrollan su carrera científica en el extranjero o fuera del ámbito universitario).
} 
Para valorar las publicaciones (35 puntos) se recurre a la clasificación de las revistas en cuatro categorías de valor decreciente (A, B, C y D). Para el campo de las Ciencias Sociales y Jurídicas se especifica que las publicaciones del tipo A serán aquellas revistas indexadas en el Journal Citacion Reports (JCR) del Social Sciences Citation Index (SSCI) y situadas en el primer y segundo cuartil de su categoría.

Las consideradas del tipo B son aquellas revistas indexadas en el Journal Citacion Reports (JCR) del Social Sciences Citation Index (SSCI) y situadas dentro del tercer y cuarto cuartil de su categoría, y en la base Impacto de las Revistas Españolas de Ciencias Sociales (IN-RECS) dentro del primer cuartil de su categoría. También se incluyen aquellas revistas recogidas en el catálogo de Difusión y Calidad Editorial de las Revistas Españolas de Humanidades y de Ciencias Sociales y Jurídicas (DICE) y aquellas recogidas en Latindex que cumplan un mínimo de 31 criterios.

Las revistas de tipo C son aquellas situadas en el segundo y tercer cuartil de su categoría en el IN-RECS y los publicados en las revistas recogidas en el catálogo DICE como revistas tipo C y en Latindex. La categoría D está pensada para aquellas revistas indexadas en otras bases de datos no mencionadas. Se remarca que en ninguna área de conocimiento será posible superar la nota de corte únicamente con artículos de esta última categoría.

En relación a las patentes, únicamente se van a valorar en este epígrafe aquellas que están en explotación entendiendo éstas como "un trabajo de excelencia con el valor añadido de trasferencia al sector productivo".

Y por último, en el apartado de otras publicaciones científicas (libros y capítulos de libros), se limita a 12 puntos la puntuación máxima a alcanzar. Los criterios de valoración se centran en el prestigio de la editorial/editores, la naturaleza de la obra y de la colección, las citas, reseñas en revistas científicas especializadas, traducciones $\mathrm{y}$ "todo ello, dentro del contexto específico en el que se ha realizado la investigación cuya productividad se está evaluando".

\subsection{A Axencia para a Calidade do Sistema Universitario de Galicia (ACSUG)}

La ACSUG evalúa el currículum del solicitante en cinco bloques de méritos: (1) Actividad investigadora (45 puntos sobre 100), (2) Actividad docente (30 puntos sobre 100), (3) Formación académica (10 puntos sobre 100), (4) Actividad profesional (10 puntos sobre 100) y (5) Experiencia en gestión (5 puntos sobre 100). La puntuación a alcanzar es de 55 puntos, siendo obligatorio poseer una puntuación mínima en dos de los apartados: (1) Actividad investigadora (5 puntos) y (2) 
La (1) Actividad investigadora se valora en función de seis parámetros, sin determinar cuál es la puntuación máxima para cada uno de ellos (a excepción de las estancias, proyectos y el apartado de otros méritos de investigación). Dicha agencia establece una horquilla de puntuación para cada uno de los méritos aportados. Los parámetros de evaluación son los siguientes: (1.1) Resultados de la actividad investigadora, en los que aparecen englobadas las publicaciones científicas, libros, congresos y conferencias y seminarios; (1.2) Proyectos y contratos de investigación; (1.3) Transferencia de los resultados; (1.4) Tesis doctorales dirigidas; (1.5) Movilidad y (1.6) Otros méritos de investigación.

En el primer epígrafe (1.1) Resultados de la actividad investigadora se distingue entre: Publicaciones científicas indexadas/no indexadas, libros y capítulos de libros, congresos y conferencias y seminarios impartidos. La clasificación de las publicaciones científicas indexadas a la que se atiende es la siguiente: Grupo A (33\% superior JCR), Grupo B (33\% central JCR), Grupo C (33\% inferior JCR), y grupo D (consideradas como no indexadas por no estar incluidas en el JCR). Observamos cómo en el protocolo de evaluación no se mencionan otros índices de calidad en función del área de conocimiento. Lo mismo ocurre en el caso de los libros y capítulos de libros, donde no se señala ningún índice de calidad sino que solamente se distingue si el autor es coordinador/autor de un libro completo o de un capítulo de libro. Cabe destacar que en esta agencia sí se hace referencia expresa al número de autores que determinará la puntuación alcanzada 22 en los méritos relativos a los resultados de la actividad investigadora.

En relación a los proyectos y contratos de investigación se diferencia entre la figura de investigador responsable y la de colaborador. En este último caso se limita la puntuación a un máximo de 0,5 por año y se establece un máximo de tres puntos.

En cuanto a la transferencia de los resultados se mencionan dos tipos de méritos: las patentes (únicamente podrán ser puntuadas con la máxima nota aquellas que están en explotación), y la transferencia de conocimiento. Por otra parte, para valorar la dirección de las tesis doctorales defendidas se tiene en cuenta la mención de calidad del programa de doctorado o la mención europea de la tesis, y si ha sido una codirección se dividirán los puntos entre el número de directores.

Esta agencia señala un apartado específico para la movilidad, entendida como estancias en centros de investigación, limitada a cinco puntos como puntuación máxima. El apartado de otros méritos de investigación también estará limitado a tres puntos. 
En cuanto a la evaluación de (2) la Actividad docente, sí se mencionan las puntuaciones máximas de valoración de cada uno de los apartados: (2.1) Dedicación docente (puntuación máxima 33 puntos), (2.2) Calidad de la actividad docente (puntuación máxima 5 puntos), (2.3) Formación didáctica para la actividad docente (puntuación máxima 3 puntos) y (2.4) Otros méritos docentes (puntuación máxima 2 puntos). Cabe señalar la prevalencia de la docencia universitaria oficial (valorada con un 0,2 por crédito impartido) frente a la docencia no universitaria impartida (puntuada con 0,25 por curso académico). Se incluye en dicho apartado la dirección de proyectos de fin de carrera, tesinas, DEA, etc...

En relación a (2.2) la calidad de la actividad docente, se valora la evaluación de la actividad a través del programa Docentia (5 puntos: muy favorable; 3 puntos: favorable) o el resultado positivo de las encuestas al alumnado (igual o superior al $70 \%$ valorado con 0,2 por materia). También se evalúa la elaboración del material docente y la participación en proyectos de innovación docente y empleo de nuevas tecnologías, así como la impartición/asistencia de cursos y seminarios de formación docente (con un mínimo de 10 horas) y las estancias en centros docentes.

\subsection{La Agenciade Calidad, Acreditación yProspectivadelas Universidades de Madrid (ACAP)}

La Agencia de Calidad, Acreditación y Prospectiva de las Universidades de Madrid establece el sistema de evaluación en función de cuatro criterios que no son los habituales hasta el momento: (1) Experiencia (45 puntos), (2) Producción académica y científica y su difusión (20 puntos), (3) Formación (15 puntos) ${ }^{23}$, y (4) Otros méritos (15 puntos). Éstas son las puntuaciones máximas directas que el solicitante puede alcanzar en cada uno de los epígrafes. Posteriormente dicha puntuación se escalará sobre 100 de tal modo que las puntuaciones quedarán así: (1) Experiencia docente, investigadora y profesional $(40 \%)$, (2) Producción académica y científica y su difusión (40\%), (3) Formación (15\%) y Otros méritos (5\%).

Cabe destacar que se valoran los méritos de los últimos diez años (a excepción del apartado de formación académica). Por otra parte también merece ser señalado el hecho de que el comité evaluador podrá aumentar la puntuación de cada uno de los apartados "en función de la apreciación cualitativa" de los méritos del solicitante. 
El primer criterio de valoración (1) Experiencia docente, investigadora y profesional "se considera como el conjunto de actividades docentes, investigadoras y profesionales desarrolladas a lo largo de su carrera." En dicho apartado se valora (1.1) Experiencia docente, (1.2) Experiencia investigadora y (1.3) Experiencia profesional.

En el apartado (1.1) Experiencia docente se citan los méritos que van a ser considerados y el cómputo de cada uno de ellas. Se diferencia entre la impartición de asignaturas en enseñanza reglada como profesor contratado (en cualquiera de las modalidades en titulaciones oficiales puntuando 0,2 puntos por créditos de 10 horas) y como profesor contratado en plantilla fuera de la Universidad (hasta 0,4 por año académico). Se debe señalar que también se valora la impartición de asignaturas de enseñanza reglada con sustancial diferencia de programa entre ellas.

También se diferencia entre la colaboración en tareas docentes en la universidad o centros universitarios (como becario, tutor Uned), la impartición de clases en máster o cursos de especialización de posgrado (no oficiales), la dirección académica de trabajos (valoración máxima de un punto), los proyectos de innovación y mejora docente (valoración máxima de dos puntos), las encuestas de alumnos con evaluaciones positivas por encima del 70\% (valoración máxima de dos puntos).

El apartado relativo a la experiencia investigadora (1.2) se evalúa en función de los proyectos de investigación y de las tesis doctorales defendidas y aprobadas. Se tiene en cuenta si el proyecto ha sido financiando externamente (hasta ocho puntos), por la universidad o institución del investigador (cuatro puntos) y también la dirección de los mismos, siempre que el equipo investigador esté compuesto por cuatro miembros mínimo (dos puntos por proyecto).

El último punto de este apartado está orientado a la (1.3) Experiencia profesional (fuera de la Universidad y relacionada con la formación académica del solicitante) valorándose las actividades en puestos técnicos o de dirección fuera de la universidad (hasta tres puntos por año) y colaboraciones puntuales o informes profesionales (hasta dos puntos por informe).

El segundo apartado (2) Producción académica y científica y su difusión se define como "la generación de conocimiento fruto de las actividades del profesorado y su puesta a disposición de terceros. Son documentos editados sobre diversos soportes y destinados a la difusión de la producción académica y científica". Se valoran las (2.1) Publicaciones, (2.2) Medios de comunicación audiovisual, (2.3) Exposiciones, contribuciones a encuentros científico-profesionales, (2.4) Documentos científicotécnicos registrados en la propiedad industrial o intelectual y (2.5) Otros méritos de 
recogido en el JCR o análogo) estableciendo tres categorías en función del tercio en el que se encuentre la publicación: A (tercio superior), B (tercio medio) y C (tercio inferior). Las consideradas sin índice de impacto son aquellas que responden a criterios similares a los incluidos en la base de datos Latindex y son catalogadas como revistas del grupo D.

Con respecto a los libros y capítulos de libro se diferencia entre los docentes (manuales, cuadernos de prácticas, libros de texto), de referencia o consulta, de investigación y la propia tesis doctoral siempre que haya sido publicada por una entidad diferente a la de la Universidad de lectura. Los más valorados son los manuales y libros de texto universitarios, los de investigación, la tesis doctoral cuando cumpla el criterio mencionado, y las traducciones y ediciones críticas de libros (hasta cuatro puntos).

También se tienen en cuenta las traducciones y ediciones críticas de libros, los capítulos de libros (limitado a dos puntos y medio) y la publicación de ponencias y comunicaciones completas (limitado a tres puntos). Un aspecto a señalar es que la puntuación se corregirá en función del número de autores (a partir de cuatro en artículos y libros) y a partir de dos para los capítulos de libro.

En el segundo apartado (2.2) Medios de comunicación audiovisual, se valoran las producciones visuales (gráficas y fotográficas), sonoras (musicales y radiofónicas) y audiovisuales (cinematográficas, televisivas y multimedia).

El epígrafe destinado a las (2.3) Exposiciones está relacionado con las artes plásticas y se distingue entre artísticas/científicas. En relación a (2.3) los documentos registrados de propiedad industrial (patentes, modelos de utilidad, obtenciones vegetales, topografías de productos semiconductores, diseño industrial, dibujos y modelos industriales) e intelectual se valora hasta dos puntos por mérito y dos puntos más si se llega a comercializar (diseños arquitectónicos y de ingeniería, mapas y diseños topográficos, programas informáticos, bases de datos y modelos de medición de intangibles). Y por último se menciona el apartado de (2.5)

Otros méritos de producción académica y científica entre los que se recogen las páginas web de las asignaturas que incluyan material, las recensiones y prólogos y los pósters y presentaciones orales a congresos (publicadas como resumen).

\subsection{Euskal Unibertsitate Sistemaren Kalitatea Ebaluatu eta Egiaztatzeko Agentzia (UNIBASQ)}


La Ley 3/2004, de 25 de febrero, del Sistema Universitario Vasco fija cuáles son los requisitos previos para poder acceder a dichas figuras. En el caso de profesor agregado tres son los requisitos "a) Estar en posesión del título de doctora o doctor; b) Acreditar, al menos, tres años de actividad docente universitaria e investigadora y c) Disponer de la evaluación positiva de la actividad docente e investigadora, que se fijará reglamentariamente por el Gobierno, acreditada con el informe de la Agencia de Evaluación de la Calidad y Acreditación del Sistema Universitario Vasco u organismo similar reconocido por la normativa vasca."

Los apartados de evaluación que establece dicha agencia son cinco valorados sobre un máximo de 100 puntos: (1) Actividad investigadora y transferencia del conocimiento (55 puntos), (2) Actividad docente y experiencia profesional (35 puntos), (3) Gestión y administración (5 puntos), (4) Formación académica adicional (5 puntos) y (5) Euskera (10 puntos). La evaluación positiva para dicha figura se obtiene si el solicitante alcanza "más de 65 puntos en total con un mínimo de 30 puntos en el apartado de Actividad Investigadora y Transferencia de Conocimiento y un mínimo de 20 puntos en el apartado de Actividad Docente y Experiencia Profesional". De nuevo observamos cómo la actividad investigadora recibe la mayor puntuación seguida de la actividad docente. Veamos ahora por separado dichos apartados.

En el epígrafe (1) Actividad investigadora y transferencia del conocimiento se valoran cinco tipos de méritos: (1.1) Publicaciones (hasta 40 puntos), (1.2) Proyectos y Contratos de Investigación/Transferencia de conocimiento (relacionada con el área y vinculada con la Universidad a través de la OTRI/DEIKER o centros similares)/Exposiciones valoradas por expertos o expertas o Intervenciones arqueológicas (hasta 15 puntos), (1.3) Congresos (hasta 5 puntos), (1.4) Actividades de Tercer Ciclo (hasta 20 puntos), (1.5) Otros méritos de investigación (hasta 3 puntos).

El apartado de publicaciones vuelve a ser el más valorado, no obstante, no se mencionan los índices de calidad que se van a tener en cuenta a la hora de evaluar. Por ejemplo, en el caso de los artículos, únicamente se señala que sean "Publicaciones Científicas Indexadas en revistas de nivel medio/alto de su área". Cabe destacar también el peso que adquieren las actividades de tercer ciclo, en las que se incluyen la dirección de tesis defendidas y también la experiencia docente universitaria de posgrado de investigación o de programas de doctorado (en Universidades u otros Organismos). 
En el apartado orientada a la (2) Actividad docente y la experiencia profesional se evalúan cuatro tipos de méritos: (2.1) Trayectoria Docente Universitaria (hasta 25 puntos), (2.2) Publicaciones docentes (hasta 10 puntos), (2.3) Innovación docente y otros méritos docentes (hasta 10 puntos) y (2.4) Experiencia Profesional (hasta 15 puntos). En este apartado, igual que en el anterior, tampoco se menciona la horquilla de puntuación y los únicos límites de puntuación son los señalados. Cabe destacar que la experiencia profesional siempre será la relacionada con el área en cuestión.

\subsection{Agència Valenciana d'Avaluació i Prospectiva (AVAP)}

La Agencia Valenciana de Evaluación y Prospectiva señala cuatro apartados de evaluación en los que la dimensión que tiene más relevancia vuelve a ser nuevamente la investigación: (1) Formación académica y experiencia profesional (20\%), (2) Experiencia investigadora y de transferencia del conocimiento (55\%), (3) Experiencia docente (20\%) y (4) Otros méritos (5\%). La puntuación mínima para alcanzar la evaluación positiva es de 55 puntos, ponderados todos los apartados. No obstante, es necesario cumplir una doble condición para alcanzar la evaluación positiva: a) alcanzar un mínimo de 20 puntos en experiencia investigadora y de transferencia del conocimiento y b) conseguir un mínimo de 15 puntos en experiencia docente.

En esta agencia se presta una mayor atención a la adaptación de la valoración de los méritos en función de las particularidades de cada una de las áreas de conocimiento. Se establecen cinco comités de evaluación (1. Ciencias sociales y Jurídicas, 2. Ciencias experimentales, 3. Ciencias de la salud, 4. Enseñanzas técnicas y 5. Humanidades), a partir de los cuales se determina la puntuación máxima que se puede obtener en cada uno de los apartados. Nuestro interés está centrado en las puntuaciones establecidas por el primero de los comités.

En el apartado (2) Experiencia investigadora y transferencia del conocimiento se establecen ocho sub-apartados a valorar, indicando las puntuaciones máximas que pueden alcanzarse en cada uno de ellos. El primero de ellos, (2.1) Artículos en revistas científicas, es el que ostenta la mayor puntuación (29 puntos).

Se explica que se valoran aquellos con "proceso anónimo de revisión por pares Peer Review, aceptándose como tales las que ocupen posiciones relevantes en los listados por ámbitos científicos en el «Subject Category Listing» del Journal Citation Reports del Science Citation Index, del Social Sciences Citation Index y del Arts and Humanities Citation Index (Institute of Scientific Information, -ISI- Philadelphia, PA, 
«Subject Category Listing» del JCR (Grupo A: primer tercio; Grupo B: segundo tercio; Grupo C: tercer tercio; Grupo D: revistas sin indexar de reconocido prestigio en el área de conocimiento con proceso anónimo de revisión por pares).

Para llegar a alcanzar la puntuación máxima será necesario que el solicitante haya publicado 10 artículos, aunque se señala que se podrán considerar un número menor en función de la calidad de las mismas en el área. Por otra parte, debemos destacar que las bases de datos DICE y RESH son mencionadas como referencia de calidad en el campo de las Ciencias Jurídicas y no de las Ciencias Sociales como es lo habitual.

En el epígrafe (2.2) Libros y capítulos de libros de investigación (11 puntos) se valoran los publicados con ISBN "en editoriales especializadas de reconocido prestigio en el correspondiente campo científico, en las que se pueda garantizar un riguroso proceso de selección y evaluación de los originales".

Se puntualiza que se tendrá en cuenta el número de autores pero no se indica el modo en el que se efectuará.

Otro apartado será el destinado a las (2.3) Tesis Doctorales y trabajos académicos dirigidos, especificando la consideración de aquellas que hayan tenido la mención de calidad y mención de doctorado europeo o la mención de calidad en el programa de doctorado. Con respecto a las (2.4) Estancias postdoctorales (formativas o de investigación) se valoran aquellas realizadas en centros españoles/extranjeros diferentes al de defensa o desarrollo de la tesis doctoral. Para su valoración se tiene en cuenta la duración y la calidad del programa o de la institución.

Por otra parte, en relación a los (2.5) Proyectos de investigación (valorados con 4,5 puntos) se señalan los siguientes criterios de evaluación: "la participación en proyectos de investigación subvencionados en convocatorias públicas y en concurrencia competitiva, en especial los financiados mediante programas nacionales, europeos $\mathrm{u}$ otros de ámbito internacional". También se consideran los financiados por comunidades autónomas $u$ entidades públicas y privadas siempre que fueran objeto de proceso de evaluación externa.

La puntuación variará en función del tipo de participación y grado de responsabilidad de la persona solicitante (investigadora o investigador principal del proyecto o como miembro del equipo de investigación). Se incluyen en este apartado también los contratos de investigación de "especial relevancia con empresas, con la administración pública, o con otras entidades". Cabe destacar que los premios de investigación v de creación artística (2.6) son valorados en un apartado específico (1,5 
propiedad intelectual (número de diseños arquitectónicos y de ingeniería, mapas y diseños topográficos, programas informáticos, bases de datos, modelos de medición intangibles y otros registros de la propiedad intelectual). No obstante no se especifican los criterios que se van a aplicar para su evaluación.

Y como último aspecto a juzgar en el apartado de la experiencia investigadora está el sub-apartado de las (2.8) contribuciones en congresos (3,3 puntos) donde se tiene en cuenta cualquier contribución (congresos, conferencias, seminarios, reuniones científicas) realizada en España/extranjero con procesos selectivos de admisión de trabajos, prestando especial atención a "la participación en comités científicos, las comunicaciones y los pósters admitidos".

Pasaremos ahora a estudiar la política que se sigue en relación a la evaluación docente, valorada con 32 puntos. Los méritos a tener en cuenta se clasifican en cuatro apartados, estableciendo una puntuación máxima en cada uno de ellos. El primero de ellos es el que hace referencia a la (3.1) Actividad docente universitaria de primer ciclo, segundo ciclo, máster oficial y doctorado en centros españoles o extranjeros (valorada con 21 puntos).

En este apartado también se tienen en cuenta los cursos impartidos en la docencia reglada no universitaria (3 puntos). Para obtener la puntuación máxima en este apartado se precisan un máximo de 45 créditos de docencia universitaria. Se valoran también las evaluaciones positivas sobre la calidad de la docencia con nota igual o superior a 7 sobre 10 o equivalente.

Otro apartado (3.2) es el de la participación en proyectos de innovación y mejora docente (máximo 2,1 puntos) subvencionados por entidades públicas o privadas en concurrencia competitiva y en planes/equipos relacionados con el EEES, sobre todo en relación a la adaptación a las competencias necesarias para el aprendizaje del alumnado.

También se valoran (3.3) las publicaciones de material docente original (libros y capítulos de libros docentes y divulgativos publicados con ISBN, según el número de autores, cualquiera que sea el soporte utilizado y otras publicaciones relacionadas con la innovación o mejora docente (libros, capítulos, artículos, webs, etc.). La máxima puntuación en este apartado son 2,4 puntos.

Y por último los (3.4) Cursos y seminarios recibidos o impartidos de duración igual o superior a 15 horas, cuyo objetivo sea la mejora de la docencia. La puntuación máxima en este apartado son 1,5 puntos sobre 100. 


\section{CONCLUSIONES}

Una vez expuestos los criterios que cada una de las agencias autonómicas publican para evaluar el currículum del solicitante a la figura de contratado doctor podemos concluir lo siguiente:

En primer lugar, queda confirmado el peso de la experiencia investigadora y de la experiencia docente como las dos dimensiones básicas para el proceso de evaluación. En todas las agencias estudiadas reciben la mayor puntuación, y en concreto, será la experiencia investigadora la más relevante en el currículum del solicitante. No obstante, algunas de ellas exigen una puntuación mínima a alcanzar en cada uno de los dos apartados como requisito básico para conseguir una acreditación positiva.

Los principales méritos a tener en cuenta en la experiencia investigadora son los artículos en publicaciones periódicas indexadas. Los índices de calidad mencionados suelen ser coincidentes (ISI, IN-RECS, DICE) en el área de las Ciencias Sociales. Sin embargo, algunas de ellas sí mencionan el número mínimo de dichas publicaciones a presentar.

Por otra parte, y en líneas generales, la política de evaluación de la docencia se realiza en función de los mismos parámetros en todas las agencias autonómicas. La docencia en titulaciones oficiales recibe el mayor peso. También se debe mencionar el escaso peso que tienen las valoraciones sobre la actividad docente

Para finalizar, tras lo observado se puede concluir que las agencias autonómicas parecen utilizar los mismos criterios de evaluación en las políticas de evaluación de la investigación y la docencia. No obstante, la información orientativa ofrecida a los solicitantes para la evaluación de los méritos difiere considerablemente de una a otra.

\section{REFERENCIAS}

Carreras, J. (2010). Evaluación de la calidad docente y promoción del profesorado (VII). Legislación universitaria española (e): modificación de la Ley Orgánica de Universidades. Profesorado contratado permanente (2004-2008). Educación Médica, 13, (4): 205-221.

Carreras, J. (2007). Evaluación de la calidad docente y promoción del profesorado (V). Legislación universitaria española (c): desarrollo de la Ley Orgánica de Universidades (2002-2005). Educación Médica, 10(2): 69-85. 
haremos mejor en el futuro. De la UCUA a la AGAE diez años de evaluación XXI. Revista de Educación, 11: 13-27.

Ley 3/2004, de 25 de febrero, del Sistema Universitario Vasco (Boletín Oficial del País Vasco, núm. 50, 12 de marzo de 2004, p.1-62).

Ley Orgánica 6/2001, de 21 de diciembre, de Universidades (Boletín Oficial del Estado, 21 diciembre 2001, núm. 307, p. 49400-49425).

Ley Orgánica 4/2007, de 12 de abril, por la que se modifica la Ley Orgánica 6/2001, de 21 de diciembre, de Universidades (Boletín Oficial del Estado, 13 abril 2007, núm. 89, pp.16241-16260).

Ley 1/2003, de 19 de febrero, de Universidades de Cataluña (Boletín Oficial del Estado, núm. 60, 11 marzo 2003, p.9454-9522).

Resolución de 26 de julio de 2005, de la Dirección General de Universidades, por la que se establecen los criterios de acreditación para las figuras contractuales de Profesorado Universitario (Boletín Oficial de la Junta de Andalucía, 8 de agosto 2005, núm. 153, p.7-9).

Tejedor, F. J.; Jornet, J. M. (2008). La evaluación del profesorado universitario en España. Revista Electrónica de Investigación Educativa, Especial. Recuperado el 23 de octubre de 2010, de http:/ / redie.uabc.mx/NumEsp1/contenidotejedorjornet.Html

\section{Natalia Quintas Froufe}

Licenciada en Publicidad y Relaciones Públicas por la Universidad de Vigo. Doctora por la Universidad de Vigo. Profesora ayudante en la Universidad de Alicante (20012010) y actualmente profesora ayudante doctor en la Universidade da Coruña. 